\title{
SENSIBILIZACIÓN A AEROALÉRGENOS EN UNA POBLACIÓN PEDIÁTRICA PERUANA CON ENFERMEDADES ALÉRGICAS
}

\author{
David García-Gomero (iD 1,2,a , María del Carmen López-Talledo (iD) 1,3,b, \\ César Galván-Calle (iD) 1,3,b , Ricardo Muñoz-León (DD) 1,3,c , Edgar Matos-Benavides (iD) 1,3,b, \\ Crhistian Toribio-Dionicio (iD) 1,2,a , Wilmer Córdova-Calderón (iD) 1,3,b \\ Centro de Referencia Nacional de Alergia, Asma e Inmunología, Instituto Nacional de Salud del Niño, Lima, Perú. \\ 2 Facultad de Medicina, Universidad Nacional Mayor de San Marcos, Lima, Perú. \\ Sociedad Peruana de Inmunología, Lima, Perú. \\ ${ }^{a}$ Médico cirujano; ${ }^{b}$ médico inmunólogo clínico alergólogo; ${ }^{\mathrm{c}}$ médico pediatra.
}

\section{RESUMEN \\ Con el objetivo de determinar el perfil de sensibilización a aeroalérgenos en pacientes pediátricos con asma y/o rinitis alérgica se realizó un estudio transversal en 411 pacientes usando una prueba cutánea de alergia. Se analizó la edad, sexo, nivel de IgE total y eosinófilos. La edad media fue de 8,1 $\pm 3,7$ años y el $60,6 \%$ fueron varones. La media de los eosinófilos y el nivel de IgE total fue de 450,1 $\pm 377,3$ células $/ \mathrm{mm}^{3}$ y $861,7 \pm 757,6 \mathrm{IU} / \mathrm{mL}$, respectivamente. Los ácaros fueron los alérgenos sensibilizantes más frecuentes (79,8\%) y el Dermatophagoides farinae $(65,2 \%)$ fue el más común. La polisensibilización estuvo presente en el 76,2\% de los pacientes. La sensibilización se evidenció en la mayoría de los pacientes con asma y/o rinitis alérgica y estuvo asociada con la edad, grupos etarios y nivel de IgE total. \\ Palabras clave: Rinitis alérgica; Asma; Alérgenos; Hipersensibilidad mediada por IgE; Perú (fuente DeCS BIREME). \\ SENSITIZATION TO AEROALLERGENS IN A PERUVIAN PEDIATRIC POPULATION WITH ALLERGIC DISEASES}

\begin{abstract}
In order to determine the profile of sensitization to aeroallergens in pediatric patients with asthma and / or allergic rhinitis, a cross-sectional study was carried out in 411 patients using an allergy skin test. Age, sex, total IgE level and eosinophils were analyzed. The mean age was $8.1 \pm 3.7$ years and $60.6 \%$ were male. The average of the eosinophils and the level of total IgE was $450.1 \pm 377.3$ cells / $\mathrm{mm}^{3}$ and $861.7 \pm 757.6$ IU / mL, respectively. Mites were the most frequent sensitizing allergens (79.8\%) and Dermatophagoides farinae (65.2\%) was the most common. Polysensitization was present in $76.2 \%$ of patients. Sensitization was evident in the majority of patients with asthma and / or allergic rhinitis and was associated with age, age groups and total IgE level.
\end{abstract}

Citar como: García-Gomero D, López-Talledo MDC, Galván-Calle C, Muñoz-León R, Matos-Benavides E, Toribio-Dionicio C, et al. Sensibilización a aeroalérgenos en una población pediátrica peruana con enfermedades alérgicas. Rev Peru Med Exp Salud Publica. 2020;37(1):5762. Doi: https://doi.org/10.17843/ rpmesp.2020.371.4460

Correspondencia: Wilmer Córdova-Calderón; Centro de Referencia Nacional de Alergia, Asma e Inmunología, Instituto Nacional de Salud del Niño; avenida Brasil 600. Lima, Perú; wilmer.cordova@gmail.com

\section{INTRODUCCIÓN}

El asma y la rinitis alérgica (RA) son las enfermedades inflamatorias crónicas más importantes en niños, con un significativo efecto económico y en la calidad de vida de estos pacientes ${ }^{(1-3)}$. La prevalencia de estas enfermedades está aumentando a nivel mundial, posiblemente por factores ambientales, dieta o cambio climático ${ }^{(1)}$. En base a diferentes estudios realizados en Perú, el asma y la RA tienen una prevalencia mayor al 30\% ${ }^{(4,5)}$, constituyéndose como las enfermedades crónicas no infecciosas más frecuentes en la edad pediátrica.

Los aeroalérgenos son los principales desencadenantes de las enfermedades alérgicas y pueden ser intra o extradomiciliarios ${ }^{(1)}$. Durante los dos primeros años, los niños están expuestos a aeroalérgenos intradomiciliarios (ácaros del polvo, hongos, partículas o excreciones de animales) mientras que, a partir de los cinco años, están expuestos a los extradomiciliarios (pólenes, hongos, grass) ${ }^{(1-3,6)}$. La exposición temprana y la presencia de atopia en pacientes 
con asma y/o RA contribuyen a la persistencia de estas enfermedades en la adultez ${ }^{(1-3)}$. Los aeroalérgenos sensibilizantes pueden variar entre países; sin embargo, su identificación es importante para un mejor abordaje clínico ${ }^{(1-3)}$.

La atopia es la tendencia personal o familiar de producir anticuerpos inmunoglobulina $\mathrm{E}$ (IgE) específicos en respuesta a la sensibilización a alérgenos ${ }^{(7)}$. Su diagnóstico se establece mediante una historia clínica compatible y se apoya en la realización de pruebas cutáneas (prick test) o pruebas in vitro que confirman la sensibilización o presencia de IgE contra determinados alérgenos ${ }^{(1-3)}$. Las pruebas cutáneas son las más usadas para detectar reacciones mediadas por IgE en pacientes con asma y RA ${ }^{(1-3)}$, debido a que ha mostrado una mayor sensibilidad y especificidad ${ }^{(8)}$ y una menor tasa de complicaciones y molestias para el paciente ${ }^{(1-3)}$.

Debido a ello, la identificación de la sensibilidad de los pacientes con asma y/o RA a aeroalérgenos permite establecer medidas preventivas y desarrollar estrategias de tratamiento más efectivas y dirigidas, como la inmunoterapia específica contra un alérgeno, la única capaz de modificar la respuesta inmune anormal en pacientes con enfermedades alérgicas, proporcionando mejoría en los síntomas y en la calidad de vida ${ }^{(1-3)}$.

El objetivo del presente estudio es establecer el perfil de sensibilización a aeroalérgenos en niños peruanos diagnosticados con asma y/o RA debido a la importancia epidemiológica y a la necesidad de realizar intervenciones específicas en estos pacientes.

\section{EL ESTUDIO}

El estudio tuvo un diseño transversal. Los pacientes fueron reclutados desde enero del 2013 hasta diciembre del 2015 en el Centro de Referencia Nacional de Alergia, Asma e Inmunología Clínica (CERNAAI) del Instituto Nacional de Salud del Niño (INSN), centro especializado en enfermedades alérgicas e inmunológicas que recibe pacientes de todo el Perú.

La población de estudio fue de 411 pacientes que se seleccionaron a través de un muestreo censal. Se incluyeron pacientes de 2 a 17 años, con diagnóstico clínico de asma y/o RA, basado en los criterios GINA y ARIA, respectivamente ${ }^{(2,3)}$. Se excluyeron a pacientes con uso de antihistamínicos y/o corticosteroides sistémicos, al menos siete días previos a la prueba cutánea; con uso de corticoesteroides tópicos en la zona de prueba, al menos 10 días antes; en inmunoterapia, al menos seis meses antes; en tratamiento inmunorregulador (uso de inmunoglobulina endovenosa); con otras enfermedades inmunológicas (enfermedades autoinmunes y/o linfoproliferativas); con dermografismo y con piel híper o hiporreactiva.

Los datos recolectados fueron la edad (categorizado en grupos etarios: 2 a 5, 6 a 11 y 12 a 18 años), sexo, niveles de IgE total ( $\mathrm{IU} / \mathrm{mL}$ ) y número de eosinófilos en el hemograma (células $/ \mathrm{mm}^{3}$ ). A todos los pacientes se les realizó la prueba cutánea según el método Prick-Film ${ }^{\circledR}$, para determinar su

\section{MENSAJES CLAVE}

Motivación para realizar el estudio: Determinar el perfil de sensibilización de pacientes pediátricos con asma y/o rinitis alérgica usando una prueba cutánea, paso fundamental para un manejo adecuado y específico de las enfermedades alérgicas.

Principales hallazgos: La mayoría de los pacientes con asma y/o rinitis alérgica presentan sensibilización al Dermatophagoides farinae y está asociado con la edad, grupos etarios y nivel de IgE.

Implicancias: Se debe considerar nuestros resultados para complementar el tratamiento de los pacientes con asma y/o rinitis alérgica con inmunoterapia específica en base a su sensibilización.

sensibilización a alérgenos específicos. Los resultados se obtuvieron en $\mathrm{mm}^{2}$ (área de la pápula) y en $\mathrm{mm}$ (diámetro de la roncha). El método usó las recomendaciones estándar para indicar un resultado positivo ${ }^{(9)}$.

La sensibilización fue establecida como resultado positivo de la prueba cutánea contra un aeroalérgeno y la polisensibilización, contra dos o más aeroalérgenos. El perfil de sensibilización fue clasificado por grupos de aeroalérgenos: ácaros de polvo, insectos, hongos y epitelio de animales. Se aplicaron 14 aeroalérgenos (7 ácaros de polvo, 2 cucarachas, 3 hongos y epitelios de perro y gato).

Los datos fueron analizados con el programa SPSS para Windows, versión 20.0. Se utilizaron porcentajes y frecuencias para la descripción de las variables cualitativas, y la media y desviación estándar para las variables cuantitativas. La distribución de variables cuantitativas se analizó usando histogramas, gráficos de cajas y la prueba de Kolmogorov-Smirnov. Se usó la prueba de chi cuadrado para evaluar la asociación entre la sensibilización, y los grupos de edad y el género. Se usó la prueba T de Student para muestras independientes para la comparación de medias. Ambas pruebas usaron un nivel de confianza de 95\%.

Antes de realizar cualquier procedimiento, los padres o tutores legales firmaron voluntariamente un consentimiento informado. Se solicitó a los pacientes mayores de siete años que firmaran un formulario de asentimiento. El protocolo del estudio y los formularios de consentimiento y asentimiento fueron aprobados por el Comité Institucional de Ética en Investigación del INSN y estuvieron de acuerdo con los principios de la Declaración de Helsinki (Proyecto CL26-12).

\section{HALLAZGOS}

La edad media de la población fue de 8,1 \pm 3,8 años. El $60,6 \%$ era de sexo masculino; el 83,5\% tenía asma; el 91,7\%, rinitis y el $75,7 \%$, RA. De los pacientes con asma, el 69,8\% 
(287/343) tuvo una prueba cutánea positiva; asimismo, el $83,7 \%(311 / 377)$ de los pacientes con RA tuvo una prueba cutánea positiva. Existe una mayor frecuencia del fenotipo alérgico (prueba positiva) en pacientes con asma y RA (Figura 1).

La media de los eosinófilos fue de 450,1 $\pm 377,3$ células $/ \mathrm{mm}^{3}$ y la media del IgE total fue de $861,7 \pm 757,6 \mathrm{IU} / \mathrm{mL}$. En cuanto a los grupos etarios, $32,4 \%$, tenía entre 2 y 5 años; $47,9 \%$, entre 6 y 11 años; y 19,7\%; entre 12 y 18 años (Tabla 1).

\section{Perfil de sensibilización}

La sensibilización estuvo presente en el 82,7\% de los pacientes. La sensibilización se asoció con la edad, grupos etarios y niveles de IgE total (Tabla 1). Se evidencia un incremento de la frecuencia de sensibilización conforme aumenta la edad (Tabla 2). Los agentes sensibilizantes más frecuentes fueron los ácaros con un $79,8 \%$, seguidos de los insectos con un $42,3 \%$ (Tabla 2). Solamente el 6,6\% de los pacientes estuvo sensibilizado contra un solo aeroalérgeno, mientras que el $47,7 \%$ de los pacientes estaba sensibilizado contra dos a cinco aeroalérgenos, y el $28,5 \%$, contra seis o más aeroalérgenos (Tabla 3).

Dermatophagoides farinae (DF) (65,2\%), Dermatophagoides pteronyssinus (DP) (53\%) y Blomia tropicalis $(47,7 \%)$ fueron los ácaros de polvo más frecuentes, 127 pacientes (30,9\%) tuvieron una prueba cutánea positiva para los tres ácaros de polvo. La sensibilización a insectos se dio principalmente por Blatella germánica $(28,2 \%)$ y Periplaneta americana $(23,4 \%)$, mientras que la sensibilización a hongos se encontró en el 17\% de los pacientes, siendo la Alternaria alternata el agente más frecuente. Finalmente, la sensibilización a epitelios de anima-

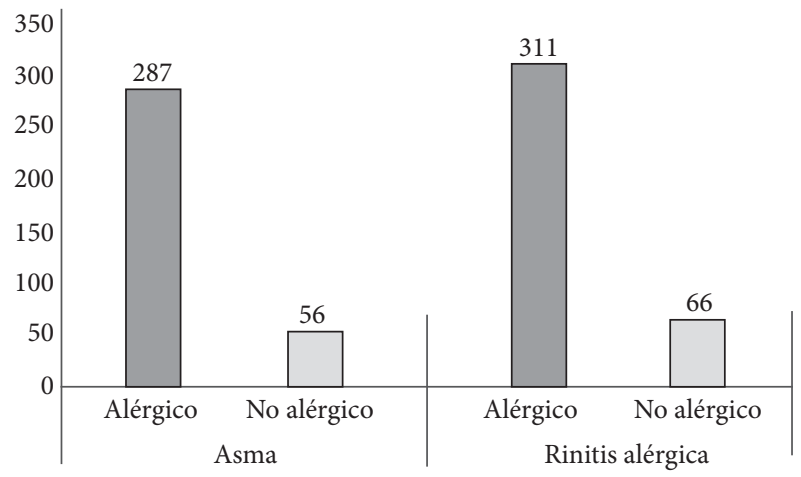

Figura 1. Fenotipo alérgico en 411 pacientes pediátricos peruanos con asma y rinitis alérgica. Centro de Referencia Nacional de Alergia, Asma e Inmunología, Lima, Perú

les se encontró en el 8,5\% de los pacientes. Para el epitelio de gato y el epitelio de perro se encontró un 6,8\% y $3,4 \%$, respectivamente (Tabla 3 ).

\section{DISCUSIÓN}

Nuestro estudio presenta por primera vez un perfil de sensibilización usando una prueba cutánea en población pediátrica peruana con diagnósticos de asma y/o RA. Se evidenció que el $82,7 \%$ de los pacientes estuvo sensibilizado a algún aeroalérgeno (76,2\% a más de uno) y se asoció con la edad, grupos etarios y niveles de IgE total. Los ácaros fueron los agentes sensibilizantes más frecuentes siendo el DF el más común $(65,2 \%)$. Se eligió la prueba cutánea por su mayor

Tabla 1. Características de 411 pacientes pediátricos peruanos con asma y/o rinitis alérgica del Centro de Referencia Nacional de Alergia, Asma e Inmunología, Lima, Perú

\begin{tabular}{|c|c|c|c|c|c|c|c|}
\hline \multirow{2}{*}{$\begin{array}{l}\text { Características } \\
\text { Edad (años), media (DE) }\end{array}$} & \multicolumn{2}{|c|}{$\begin{array}{c}\text { Total } \\
(\mathrm{n}=411)\end{array}$} & \multicolumn{2}{|c|}{$\begin{array}{c}\text { No sensibilizados } \\
(n=71)\end{array}$} & \multicolumn{2}{|c|}{$\begin{array}{c}\text { Sensibilizados } \\
\quad(\mathrm{n}=340)\end{array}$} & \multirow{2}{*}{$\begin{array}{c}\text { Valor de } \mathbf{p} \\
0,001^{\mathrm{a}}\end{array}$} \\
\hline & 8,1 & 3,8 & 6,8 & 3,5 & 8,3 & 3,8 & \\
\hline Grupos etarios (años), n (\%) & & & & & & & $0,007^{b}$ \\
\hline 2 a 5 & 133 & 32,4 & 34 & 47,9 & 99 & 29,1 & \\
\hline 6 a 11 & 197 & 47,9 & 28 & 39,4 & 169 & 49,7 & \\
\hline 12 a 18 & 81 & 19,7 & 9 & 12,7 & 72 & 21,2 & \\
\hline Género, n (\%) & & & & & & & $0,284^{\mathrm{b}}$ \\
\hline Masculino & 249 & 60,6 & 39 & 54,9 & 210 & 61,8 & \\
\hline Femenino & 162 & 39,4 & 32 & 45,1 & 130 & 38,2 & \\
\hline Asma, n (\%) & 343 & 83,5 & 56 & 78,9 & 287 & 84,4 & $0,280^{\mathrm{b}}$ \\
\hline Rinitis, n (\%) & 377 & 91,7 & 66 & 92,1 & 311 & 91,5 & $0,679^{b}$ \\
\hline Asma y rinitis, n (\%) & 314 & 76,4 & 51 & 71,8 & 263 & 77,4 & $0,278^{\mathrm{b}}$ \\
\hline Eosinófilos, media (DE) & 450,1 & 377,3 & 5,5 & 5,5 & 14,6 & 88,1 & $0,656^{\mathrm{a}}$ \\
\hline IgE, media (DE) & 861,7 & 757,6 & 338,3 & 476,07 & 961,7 & 761,8 & $<0,001^{a}$ \\
\hline
\end{tabular}

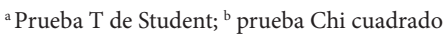

DE: desviación estándar 
Tabla 2. Perfil de sensibilización en 411 pacientes pediátricos peruanos con asma y/o rinitis alérgica del Centro de Referencia Nacional de Alergia, Asma e Inmunología. Lima, Perú

\begin{tabular}{|c|c|c|c|c|c|c|c|c|}
\hline \multirow{3}{*}{ Perfil de sensibilización } & \multicolumn{6}{|c|}{ Grupos etarios } & \multirow{2}{*}{\multicolumn{2}{|c|}{$\begin{array}{c}\text { Total } \\
(\mathrm{n}=411)\end{array}$}} \\
\hline & \multicolumn{2}{|c|}{ 2-5 años (n=133) } & \multicolumn{2}{|c|}{ 6-11 años $(n=197)$} & \multicolumn{2}{|c|}{$12-18$ años $(n=81)$} & & \\
\hline & $\mathrm{n}$ & $\%$ & $n$ & $\%$ & $\mathrm{n}$ & $\%$ & $\mathbf{n}$ & $\%$ \\
\hline Asma alérgica & 83 & 62,4 & 143 & 72,6 & 61 & 75,3 & 287 & 69,8 \\
\hline Rinitis alérgica & 92 & 69,2 & 158 & 80,2 & 61 & 75,3 & 311 & 75,7 \\
\hline Asma y rinitis alérgica & 77 & 57,9 & 135 & 68,9 & 50 & 61,7 & 263 & 64,0 \\
\hline Ácaros de polvo & 92 & 69,2 & 164 & 83,3 & 72 & 88,9 & 328 & 79,8 \\
\hline D. farinae & 79 & 59,4 & 133 & 67,5 & 56 & 69,1 & 268 & 65,2 \\
\hline D. pteronyssinus & 64 & 48,1 & 102 & 51,8 & 52 & 64,2 & 218 & 53,0 \\
\hline Blomia tropicalis & 39 & 29,3 & 100 & 50,8 & 57 & 70,4 & 196 & 47,7 \\
\hline Chortoglyphus arcuatus & 30 & 22,6 & 89 & 45,2 & 35 & 43,2 & 154 & 37,5 \\
\hline Acarus siro & 18 & 13,5 & 81 & 41,1 & 46 & 56,8 & 145 & 35,3 \\
\hline Tyrophagus putrescentiae & 25 & 18,8 & 65 & 32,9 & 31 & 38,3 & 121 & 29,4 \\
\hline Lepidoglyphus destructor & 20 & 15,0 & 64 & 32,5 & 36 & 44,4 & 120 & 29,2 \\
\hline Insectos & 49 & 36,8 & 77 & 39,1 & 48 & 59,7 & 174 & 42,3 \\
\hline Blatella germanica & 26 & 19,6 & 55 & 27,9 & 35 & 43,2 & 116 & 28,2 \\
\hline Periplaneta americana & 29 & 21,8 & 40 & 20,3 & 27 & 33,3 & 96 & 23,3 \\
\hline Hongos & 22 & 16,5 & 32 & 16,2 & 16 & 19,8 & 70 & 17,0 \\
\hline Alternaria alternata & 14 & 10,5 & 26 & 13,2 & 8 & 9,8 & 48 & 11,7 \\
\hline Aspergillus fumigatus & 7 & 5,3 & 8 & 4,1 & 7 & 8,6 & 22 & 5,4 \\
\hline Penicillium notatum & 6 & 4,5 & 4 & 2,0 & 5 & 6,2 & 15 & 3,7 \\
\hline Epitelio de animal & 11 & 8,3 & 15 & 7,6 & 9 & 11,1 & 35 & 8,5 \\
\hline Epitelio de perro & 8 & 6,0 & 12 & 6,1 & 8 & 9,9 & 28 & 6,8 \\
\hline Epitelio de gato & 5 & 3,8 & 7 & 3,6 & 2 & 2,5 & 14 & 3,4 \\
\hline
\end{tabular}

Las categorías incluidas en la tabla no son mutuamente excluyentes

sensibilidad y especificidad (>85\%) ${ }^{(7)}$ y una menor tasa de complicaciones y molestias para el paciente ${ }^{(1-3)}$. La prueba es recomendada en las guías de práctica clínica de asma y RA (GINA y ARIA, respectivamente) ${ }^{(2,3)}$.

En nuestro contexto se han llevado a cabo algunos estudios que evaluaron el perfil de sensibilización. El estudio denominado PURA (Peru Urban versus Rural Asthma) incluyó adolescentes entre 13 y 15 años de dos ciudades diferentes (una urbana y otra rural) para evaluar la prevalencia de asma y sus factores de riesgo asociados usando una prueba cutánea ${ }^{(10)}$. La prevalencia de asma y sensibilización fue de $12 \%$ y $3 \%$, y de $56 \%$ y $38 \%$ en Lima y Tumbes, respectivamente. Un análisis posterior encontró que el $61 \%$ de los sujetos con RA estuvieron sensibilizados ${ }^{(11)}$. La sensibilización fue menor en comparación con nuestro estudio y podría deberse al menor número de pacientes con asma y RA diagnosticados (84 y 22 (asma), y 166 y 95 (RA) en Lima y Tumbes, respectivamente).

Por otro lado, un estudio de casos y controles realizado en dos comunidades en Lima incluyó niños entre 9 y 19 años. De los 412 pacientes asmáticos, 315 (78,2\%) fueron atópicos ${ }^{(12)}$. Estos resultados son similares al nuestro; sin embargo, se debe resaltar que la evaluación de la atopia fue realizada usando el ImmunoCap 250 (detección sérica de IgE específica) para tres alérgenos (animal, moho y ácaros de polvo) siendo un nivel de IgE específica $>0,1 \mathrm{kU} / \mathrm{L}$ un resultado positivo. Este nivel podría explicar la alta frecuencia de atopia, ya que un punto de corte de $>0,35 \mathrm{kU} / \mathrm{L}$ es usado comúnmente en otros estudios.

Existen resultados diferentes en otros contextos. La Encuesta Nacional de Examen de Salud y Nutrición (NHANES) III, realizada en Estados Unidos entre 1988 y 1994, encontró una frecuencia de sensibilización usando una prueba cutánea en el 54,3\% de los sujetos de estudio que tenían entre 6 y 59 años de edad (población multiétnica) ${ }^{(13)}$. Por otro lado, un estudio en Marruecos, que incluyó 379 pacientes, encontró que el 3,2\% de los pacientes asmáticos fueron mono y polisensibilizados, mientras que el $18,2 \%$ y el $39,8 \%$ de los pacientes con RA fueron mono y polisensibilizados, respectivamente ${ }^{(14)}$. Un estudio realizado en España evidenció sensibilización a aeroalérgenos (midiendo IgE específica) en el $25,9 \%(121 / 468)$ de pacientes entre 0 a 5 años con sibilancias o dermatitis atópica ${ }^{(15)}$. Esta diferencia de resultados podría ser explicada por la alta prevalencia de atopia y enfermedades alérgicas en nuestro país, así como a factores ambientales como el clima, la altitud y el estado económico.

El DF fue el aeroalérgeno más común, resultados similares han sido reportados por otros autores ${ }^{(13,15)}$. Sin embargo, otros autores describen una mayor sensibilización a DP. Un 
Tabla 3. Sensibilización por número de alérgenos en 340 pacientes pediátricos peruanos con asma y/o rinitis alérgica del Centro de Referencia Nacional de Alergia, Asma e Inmunología. Lima, Perú

\begin{tabular}{|c|c|c|c|c|c|c|c|c|}
\hline \multirow{3}{*}{$\begin{array}{l}\text { Sensibilización por número de } \\
\text { alérgenos }\end{array}$} & \multicolumn{6}{|c|}{ Grupos etarios } & \multirow{2}{*}{\multicolumn{2}{|c|}{$\begin{array}{c}\text { Total } \\
(\mathbf{n}=340)\end{array}$}} \\
\hline & \multicolumn{2}{|c|}{$\begin{array}{c}2-5 \text { años } \\
(n=99)\end{array}$} & \multicolumn{2}{|c|}{$\begin{array}{c}6 \text { - } 11 \text { años } \\
(n=169)\end{array}$} & \multicolumn{2}{|c|}{$\begin{array}{c}12-18 \text { años } \\
(n=72)\end{array}$} & & \\
\hline & $\mathbf{n}$ & $\%$ & $\mathbf{n}$ & $\%$ & $\mathbf{n}$ & $\%$ & $\mathbf{n}$ & $\%$ \\
\hline Uno & 11 & 11,1 & 15 & 8,9 & 1 & 1,4 & 27 & 8,0 \\
\hline Dos a cinco & 70 & 70,7 & 93 & 55,0 & 33 & 45,8 & 196 & 57,6 \\
\hline Seis o más & 18 & 18,2 & 61 & 36,1 & 38 & 52,8 & 117 & 34,4 \\
\hline
\end{tabular}

estudio realizado en un área urbana de México encontró que este ácaro fue el alérgeno más frecuente en niños escolares con asma $(61 \%)^{(16)}$, así como en Jordania $(11,9 \%)^{(6)}$ y en Estados Unidos $(31,2 \%)^{(17)}$; estos últimos realizados en niños con asma que acudieron a un centro pulmonar pediátrico. Por otro lado, los agentes sensibilizantes más frecuentes fueron el polen de olivo (18\%) en Jordania ${ }^{(6)}$ y los pólenes de gramineas (48\%), de árboles (34\%) y de malezas (18\%) en un estudio realizado en España ${ }^{(18)}$.

Otros estudios reportan a los insectos en el segundo o tercer lugar de frecuencia, alcanzando el $21,6 \%{ }^{(19)}$, el 26,1\% ${ }^{(13)} \mathrm{o}$ el $32 \%{ }^{(17)}$. La alta sensibilización a insectos que se encuentra en nuestro estudio puede deberse a diferencias en los entornos de vivienda y comunidad ${ }^{(13)}$. En nuestro estudio, Alternaria alternata predominó en tipo de hongos. En contraste, Candida albicans y Cladosporum fueron los hongos predominantes en un estudio colombiano en el $12,9 \%$ de los pacientes ${ }^{(20)}$. La frecuencia de sensibilización fue similar para epitelio de perro y gato; resultados similares se evidencian en otros reportes ${ }^{(13,14,16-19)}$.

Las limitaciones de nuestro estudio fueron no considerar el clima donde residen nuestros pacientes, aspecto considerado y recomendado en otros estudios que encontraron una mayor frecuencia de sensibilización para pólenes y otros alérgenos ${ }^{(6,18)}$, además, solo utilizamos la prueba cutánea para evaluar la sensibilización y no el nivel de IgE específica como otros estudios ${ }^{(12,15,18)}$.

En conclusión, la mayoría de los pacientes con asma y/o RA estuvieron sensibilizados según los resultados de la prueba cutánea. El principal agente sensibilizador fue DF y la sensibilización se asoció con la edad, grupos etarios y nivel de IgE total.

Agradecimientos: Los autores agradecen a todos los participantes del estudio. También estamos agradecidos con la Oficina Ejecutiva de Apoyo a la Investigación y Docencia Especializada (OEAIDE) y con el Comité Institucional de Ética en Investigación (CIEI) del Instituto Nacional de Salud del Niño (INSN).

Contribuciones de los autores: WCC, DGG, MCLT, CGC, RML y EMB contribuyeron a la concepción y diseño del trabajo. DGG y CTD contribuyeron a la adquisición y análisis de datos y redacción del manuscrito. WCC supervisó el trabajo. Todos los autores contribuyeron a la interpretación de los datos, proporcionaron comentarios críticos y aprobaron la versión final.

Financiamiento: El apoyo financiero fue proporcionado por la Oficina Ejecutiva de Apoyo a la Investigación y Docencia Especializada (OEAIDE) del Instituto Nacional de Salud del Niño (INSN) (Proyecto CL26-12).

Conflictos de interés: Los autores declaran que no existe conflicto de intereses en relación con esta publicación.

\section{REFERENCIAS BIBLIOGRÁFICAS}

1. World Allergy Organization. WAO White Book on Allergy: Update 2013 [Internet]. Milwaukee: WAO;2013 [citado el 3 de diciembre de 2019]. Disponible en: https://www.worldallergy.org/wao-white-book-on-allergy

2. Global Initiative for Asthma. Global Strategy for Asthma Management and Prevention [Internet]. Fontana: GINA; 2019 [citado el 26 de noviembre de 2019]. Disponible en: https://ginasthma.org/2018-gina-report-global-strategy-for-asthma-management-and-prevention/

3. Bousquet J, Khaltaev N, Cruz AA, Denburg J, Fokkens WJ, Togias A, et al. Allergic Rhinitis and its Impact on Asthma (ARIA) 2008 update (in collaboration with the World Health Organization, GA(2)LEN and AllerGen). Allergy. 2008;63 Suppl 86:8-160. doi: 10.1111/j.13989995.2007.01620.x

4. Pearce N, Ait-Khaled N, Beasley R, Mallol J, Keil U, Mitchell E, et al. Worldwide trends in the prevalence of asthma symptoms: phase III of the International Study of Asthma and Allergies in Childhood (ISAAC). Thorax. 2007;62(9):758-66. doi: 10.1136/thx.2006.070169

5. Björkstén B, Clayton T, Ellwood P, Stewart A, Strachan D, ISAAC Phase III Study Group. Worldwide time trends for symptoms of rhinitis and conjunctivitis: Phase III of the International Study of Asthma and Allergies in Childhood. Pediatr Allergy Immunol. 2008;19(2):110-24. doi: 10.1111/j.1399-3038.2007.00601.x

6. Al-Zayadneh EM, Alnawaiseh NA, Altarawneh AH, Aldmour IH, Albataineh EM, Al-Shagahin $\mathrm{H}$, et al. Sensitization to inhaled allergens in asthmatic children in southern Jordan: a cross-sectional study. Multidiscip Respir Med. 2019;14:37. doi: 10.1186/s40248-019-0199-y

7. Johansson SGO, Bieber T, Dahl R, Friedmann PS, Lanier BQ, Lockey $\mathrm{RF}$, et al. Revised nomenclature for allergy for global use: Report of the Nomenclature Review Committee of the World Allergy Organization, October 2003. J Allergy Clin Immunol. 2004;113(5):832-6. doi: 10.1016/j.jaci.2003.12.591

8. Tschopp JM, Sistek D, Schindler C, Leuenberger P, Perruchoud AP, Wüthrich B, et al. Current allergic asthma and rhinitis: diagnostic efficiency of three commonly used atopic markers (IgE, skin prick tests, and Phadiatop). Results from 8329 randomized adults from the SAPALDIA Study. Swiss Study on Air Pollution and Lung Diseases in Adults. Allergy. 1998;53(6):608-13. doi: 10.1111/j.1398-9995.1998.tb03937.x 
9. Dreborg S, Backman A, Basomba A, Bousquet J, Dieges P, Malling HJ. Skin tests used in type I allergy testing Position paper. Sub-Committee on Skin Tests of the European Academy of Allergology and Clinical Immunology. Allergy. 1989;44 Suppl 10:1-59.

10. Robinson CL, Baumann LM, Gilman RH, Romero K, Combe JM, Cabrera L, et al. The Peru Urban versus Rural Asthma (PURA) Study: methods and baseline quality control data from a cross-sectional investigation into the prevalence, severity, genetics, immunology and environmental factors affecting asthma in adolescence in Peru. BMJ Open. 2012;2(1):e00421. doi: 10.1136/bmjopen-2011-000421

11. Baumann LM, Romero KM, Robinson CL, Hansel NN, Gilman RH, Hamilton RG, et al. Prevalence and risk factors for allergic rhinitis in two resource-limited settings in Peru with disparate degrees of urbanization. Clin Exp Allergy. 2015;45(1):192-9. doi: 10.1111/cea.12379

12. Nicholson A, Pollard SL, Lima JJ, Romero K, Tarazona-Meza C, Malpartida-Guzmán G, et al. Serum folate concentrations, asthma, atopy, and asthma control in Peruvian children. Respir Med. 2017;133:29-35. doi: 10.1016/j.rmed.2017.10.026

13. Arbes SJ Jr, Gergen PJ, Elliott L, Zeldin DC. Prevalences of positive skin test responses to 10 common allergens in the US population: results from the third National Health and Nutrition Examination Survey. J Allergy Clin Immunol. 2005;116(2):377-83. doi: 10.1016/j.jaci.2005.05.017

14. Bardei F, Bouziane H, Kadiri M, Rkiek B, Tebay A, Saoud A. Skin sensitisation profiles to inhalant allergens for patients in Tétouan city (North West of Morocco). Rev Pneumol Clin. 2016;72(4):221-7. doi: 10.1016/j.pneumo.2016.04.005
15. Carvajal Urueña I, Díaz Vázquez C, Cano Garcinuño A, García Merino A, Morell Bernabé JJ, Pascual Pérez JM, et al. Perfil de sensibilización alérgica en niños de 0 a 5 años con sibilancias o dermatitis atópica. An Pediatría. 2010;72(1):30-41. doi: 10.1016/j. anpedi.2009.09.011

16. Alcalá-Padilla G, Bedolla-Barajas M, Kestler-Gramajo A, Valdez-López F. Prevalencia de sensbilización a alergenos en niños escolares con asma que viven en la zona metropolitana de Guadalajara. Rev Alerg Mex. 2016;63(2):135-42. doi: 10.29262/ram.v63i2.184

17. Nagarajan S, Ahmad S, Quinn M, Agrawal S, Manilich E, Concepcion $\mathrm{E}$, et al. Allergic sensitization and clinical outcomes in urban children with asthma, 2013-2016. Allergy Asthma Proc. 2018;39(4):281-8. doi: 10.2500/aap.2018.39.4147

18. Caro Rebollo J, Moneo Hernández MI, Cabañas Bravo MJ, Garín Moreno AL, Oliván Otal MP, Cenarro Guerrero T. Valoración del estudio alérgico en niños con atopia. Pediatría Aten Primaria. 2010;12(46):227-37.

19. Sánchez-Borges M, Capriles-Hulett A, Torres J, Ansotegui-Zubeldia IJ, Castillo A, Dhersy A, et al. Diagnosis of allergic sensitization in patients with allergic rhinitis and asthma in a tropical environment. Rev Alerg Mex. 2019;66(1):44-54. doi: 10.29262/ram.v66i1.570

20. Bissinger I, Bareño J. Perfil de sensibilización a hongos en Medellín, Colombia. Rev Alerg Mex. 2016;63(2):123-34. doi: 10.29262/ram. v63i2.108

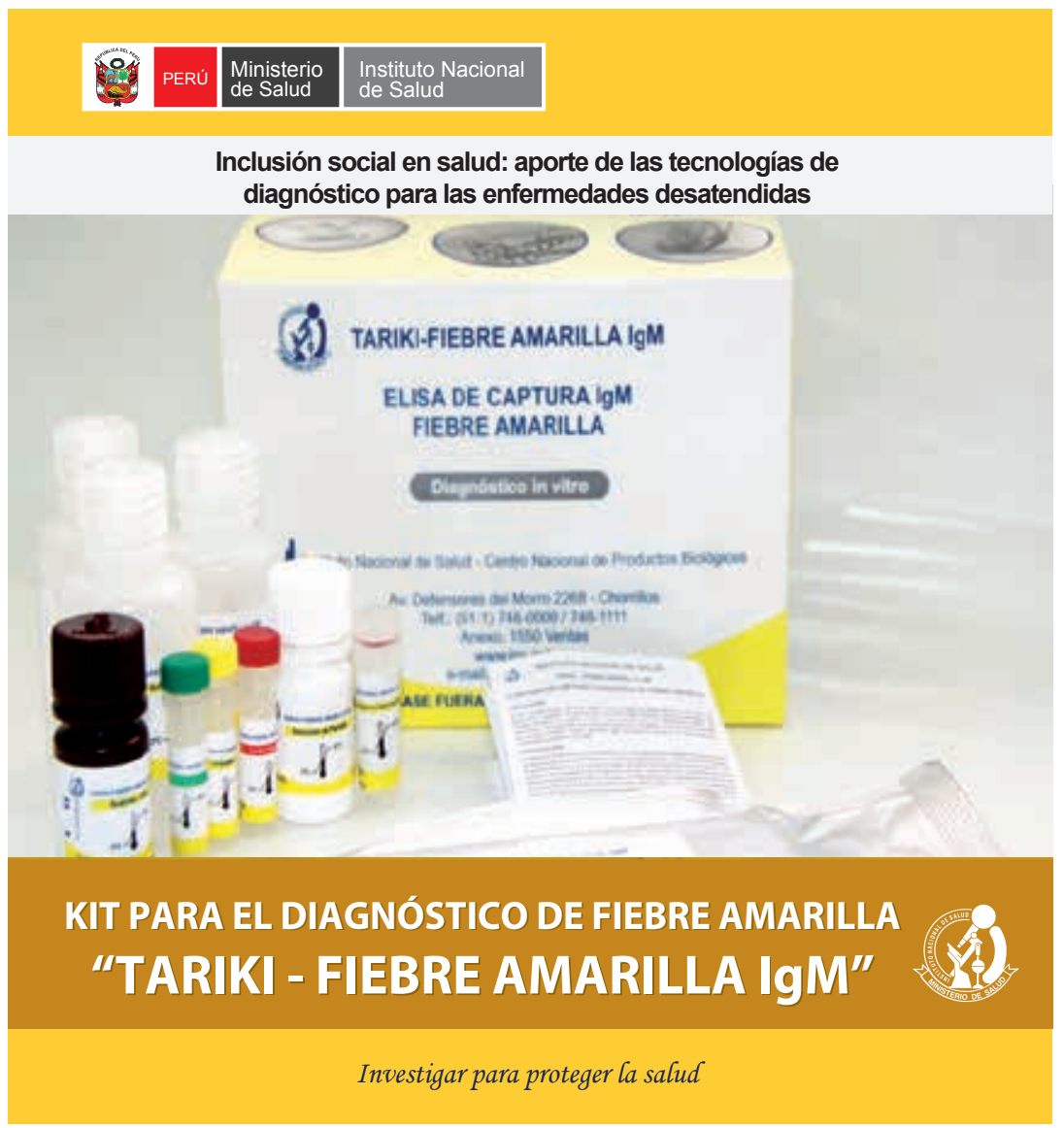

